Review Article

\title{
A Systematic Review and Meta-Analysis of Clinical Outcomes of Intravitreal Anti-VEGF Agent Treatment Immediately after Cataract Surgery for Patients with Diabetic Retinopathy
}

\author{
Li-Quan Zhao $\mathbb{D D}^{1}$ and Jin-Wei Cheng ${ }^{2}$ \\ ${ }^{1}$ Department of Ophthalmology, Pudong New Area People's Hospital Affiliated to Shanghai University of Medicine \& \\ Health Sciences, Shanghai, China \\ ${ }^{2}$ Department of Ophthalmology, Shanghai General Hospital, Shanghai Jiao Tong University School of Medicine, Shanghai, China
}

Correspondence should be addressed to Li-Quan Zhao; 526509003@qq.com

Received 3 November 2018; Revised 25 February 2019; Accepted 31 March 2019; Published 16 April 2019

Academic Editor: Manuel S. Falcão

Copyright (c) 2019 Li-Quan Zhao and Jin-Wei Cheng. This is an open access article distributed under the Creative Commons Attribution License, which permits unrestricted use, distribution, and reproduction in any medium, provided the original work is properly cited.

\begin{abstract}
Aims. To examine possible benefits of intravitreal anti-vascular endothelial growth factor (VEGF) agent treatment immediately after cataract surgery for patients with diabetic retinopathy (DR). Methods. A comprehensive literature search was performed using the Cochrane collaboration methodology to identify randomized controlled trials (RCTs) and comparative studies of cataract surgery with or without anti-VEGF agent treatment for any diabetic retinopathy. Meta-analyses were performed for clinical outcome parameters including changes in macular thickness (MT), best-corrected visual acuity (BCVA), incidence of diabetic retinopathy and maculopathy progression, laser treatment rate, and other complications. Results. Nine RCTs and 3 nonrandomized comparative studies were identified and used for comparing cataract surgery with intravitreal bevacizumab (IVB) or intravitreal ranibizumab (IVR) treatment (338 eyes, intervention group) to cataract surgery alone (329 eyes, control group). Analysis of all data showed that the mean BCVA at 1 week postoperatively had no statistically significant difference in the two groups, but at 1,3, and 6 months postoperatively, the mean BCVA was statistically significantly better in the anti-VEGF treatment group than that in cataract surgery alone group. Analysis of all data showed that the mean MT was statistically significantly less in the anti-VEGF treatment group at 1 week and 1,3 , and 6 months postoperatively $(P=0.05, P=0.006, P=0.0001$, and $P=0.0001$, respectively); but postoperative clinical outcomes were differentiated from the type of anti-VEGF agents, IVB or IVR, and the existing macular edema preoperatively. Intravitreal anti-VEGF agent treatment statistically significantly reduced the incidence of diabetic retinopathy progression and maculopathy progression compared to the control group $(P=0.0003$, $P<0.00001$, respectively). Conclusion. IVB or IVR treatment immediately after cataract surgery may represent a safe and effective strategy to prevent postoperative macular thickening or reduce macular edema and result in greater mean improvements in visual acuity for diabetic patients.
\end{abstract}

\section{Introduction}

Diabetic macular edema (DME) is a major cause of vision loss for diabetic patients $[1,2]$. Diabetic patients have a higher prevalence of early cataract need for surgery. The evidences revealed that intraocular level of inflammatory cytokines and VEGF in eyes with diabetic retinopathy can be further increased by cataract surgery and significantly increase the risk of DME, which was different from cystoid macular edema (CME) following cataract surgery (IrvineGass syndrome) for nondiabetic patients [3-5]. Topical nonsteroidal anti-inflammatory drugs (NSAIDs), either as monotherapy or combined with topical corticosteroids, appear to be effective for the prophylaxis and treatment of CME, but the intravitreal administration of anti-VEGF or steroids, at the moment of cataract surgery, seems to be a feasible solution to prevent or cure diabetic macular edema $[6,7]$. 
Intravitreal anti-VEGF agents are widely accepted as a first-line treatment for use in macular edema following central retinal vein occlusion, wet age-related macular degeneration, diabetic retinopathy, etc. [8-10]. Many studies have reported clinical outcomes of intravitreal bevacizumab (IVB) or intravitreal ranibizumab (IVR) as an adjunct to cataract surgery in the management of diabetic retinopathy (DR) progression, especially macular edema for diabetic patients [11-22]. Due to lack of systematic or larger sample size studies weighting the benefits with the possible adverse effects, the intravitreal anti-VEGF agent treatment remains questionable in diabetic patients undergoing cataract surgery.

In an attempt to detect benefits in safety and efficacy as the primary comparative criteria, we performed a systematic review and meta-analysis of existing randomized controlled trials (RCTs) and high-quality comparative studies of cataract surgery with or without intravitreal anti-VEGF agent treatment for the treatment of DR including nonproliferative diabetic retinopathy (NPDR), proliferative diabetic retinopathy (PDR), or clinically significant macular edema (CSME).

\section{Materials and Methods}

This meta-analysis was performed according to a predetermined protocol described previously [23, 24].

2.1. Literature Search. Two reviewers independently searched the following electronic databases: PubMed, EMBASE, and Wanfang Data (e-resources for China studies) and the Cochrane Controlled Trials Register up to June 30, 2018. For maximum sensitivity, we used free text and thesaurus terms including "cataract surgery or phacoemulsification," "diabetic retinopathy or diabetic macular edema," and "bevacizumab or Avastin or ranibizumab or Lucentis or aflibercept or anti-vascular endothelial growth factor agents." All published RCTs and comparative studies comparing cataract surgery alone versus cataract surgery with intravitreal anti-VEGF agent treatment for diabetic retinopathy were included. Patients preparing for cataract surgery were presented with diabetic retinopathy and greater than 18 years. At least one or more clinical outcomes representing clinical outcome parameters must be assessed and published. There was no language restriction on the publications.

The selected studies were appraised by two reviewers, who independently assessed their quality using the methods recommended in the Cochrane Handbook for Systematic Reviews of Interventions [25].

Discordance about study inclusion and quality assessment between the two reviewers was resolved through discussion until $100 \%$ agreement was reached on the final interpretation of the data.

2.2. Outcome Measure. The clinical outcome parameters included mean central macular thickness (CMT), bestcorrected visual acuity (BCVA), incidence of diabetic retinopathy and maculopathy progression, incidence of neovascular glaucoma (NVG), and rate of laser photocoagulation treatment postoperatively. Other adverse events such as elevation of intraocular pressure (IOP), retinal detachment, and ocular inflammation were all recorded.

2.3. Data Extraction and Analysis. The studies were tabulated and methodologically evaluated to assess homogeneity. Any heterogeneity between the studies would not be justified to pool the assessed outcomes. A customized data extraction form, as described in the Cochrane Handbook for Systematic Reviews of Interventions, was used to record the duration of the trial, sample size, dropouts, the system and ocular baseline features, the inclusion and exclusion criterion for patients, the dosage and location of intravitreal anti-VEGF agent injection, and postoperative treatment.

2.4. Statistical Analysis. Quantitative data were analyzed using the Cochrane Review Manager (RevMan) version 5.0 software. Summary estimates, including 95\% confidence interval (CI), were calculated. For continuous outcome data (e.g., mean macular thickness) means and standard deviations were used to calculate a weighted mean difference (WMD). For dichotomous outcomes (e.g., incidence of retinopathy progression), the odds ratio (OR) was calculated.

Statistical heterogeneity was tested using Q statistic of chi-square value test and $I^{2}$ test. Fixed effects models were used, unless significant evidence of statistical heterogeneity or clinical diversity was found. For results showing significant heterogeneity $\left(I^{2}>50 \%\right)$, random-effects meta-analysis was performed. Outcome measures were assessed on an intent-to-treat (ITT) basis. A $P$ value less than 0.05 was considered statistically significant. Subgroup analysis was performed according to the inclusion criterion.

\section{Results}

Nine RCTs and 3 nonrandomized comparative studies published between 2009 and 2016 met the inclusion criteria [11-22]. Each study revealed that there were no significant differences in preoperative demographic features, such as preoperative BCVA and macular thickness that were related to anti-VEGF treatment.

Six studies reported clinical outcomes of intravitreal bevacizumab as an adjunct to cataract surgery in the management of DME $[11-15,21]$, and 6 studies reported intravitreal ranibizumab management [16-20, 22].

Five studies included NPDR patients with no preexisting DME and evaluated intravitreal anti-VEGF agents at cataract surgery for prevention of postoperative DME $[12,16,17,19,22]$; five studies included NPDR patients with preexisting DME and evaluated intravitreal anti-VEGF agents at cataract surgery for management of diabetic maculopathy $[13,14,18,20,21]$. Two studies included NPDR or PDR patients with or without CSME and evaluated intravitreal anti-VEGF agents at cataract surgery for management of diabetic maculopathy $[11,15]$. 
Four studies reported that laser photocoagulation was performed according to ETDRS guidelines during the follow-up period $[11,12,15,16]$. Laser photocoagulation was not performed during the follow-up period in the other 8 studies [13, 14, 17-22].

Additive other treatments, such as intravitreal antiVEGF agent treatment, were not performed during the follow-up period in the all included studies.

The present meta-analysis involved 338 eyes receiving cataract surgery with anti-VEGF agent treatment and 329 eyes treated with cataract surgery alone. The selection of 12 studies is summarized in Tables 1 and 2 .

3.1. Macular Thickness. For NPDR without DME, one study [12] applying IVB revealed that the mean MT at 1 month and 6 months postoperatively was statistically significantly less in the IVB treatment group than the control group; 4 studies $[16,17,19,22]$ applying IVR revealed that the MT at 1 week and 1,3 , and 6 months postoperatively was statistically significantly less in the IVR treatment group(Table 3). All data showed that change of postoperative MT from 1 week to 6 months compared with the baseline was minor or increased little in the anti-VEGF treatment group (from -1 to $+24 \mu \mathrm{m}$ ), but there was apparent increase of postoperative MT compared with the baseline in the control group (from +12 to $+90 \mu \mathrm{m})$.

For NPDR with DME, 3 studies $[13,14,21]$ applying IVB revealed that the mean $\mathrm{MT}$ at 1,3 , and 6 months postoperatively was statistically significantly less in the IVB treatment group than the control group; 2 studies $[18,20]$ applying IVR revealed that the MT at 1 week and 1, 3, and 6 months postoperatively was statistically significantly less in the IVR treatment group (Table 3). All data showed that change of postoperative MT from 1 week to 6 months compared with the baseline was minor or decreased apparently in the anti-VEGF treatment group (from +10 to $-98 \mu \mathrm{m}$ ), but there was apparent increase of postoperative MT compared with the baseline in the control group (from -22 to $+77 \mu \mathrm{m})$.

Two studies $[11,15]$ reported the same inclusion criteria: mixed CSME, NPDR, or PDR. And the postoperative laser photocoagulations were performed based on ETDRS criterion. Analysis of these data showed that there was no statistically significant difference of the mean MT in the two groups at 1 and 3 months postoperatively (WMD: -4.34 ; 95\% CI: $-39.49,30.81 ; P=0.81$; heterogeneity: $P=0.92$, $I^{2}=0 \%$ ) (WMD: $-3.54 ; 95 \% \mathrm{CI}:-39.45,32.36 ; P=0.85$; heterogeneity: $\left.P=0.74, I^{2}=0 \%\right)$. However, at 6 months postoperatively, the mean MT was statistically significantly less in the IVB treatment group (WMD: -40.18 ; 95\% CI: -77.01, -3.36; $P=0.03$; heterogeneity: $\left.P=0.64, I^{2}=0 \%\right)$.

Analysis of all data showed that the mean MT was statistically significantly less in the anti-VEGF treatment group at 1 week and 1, 3, and 6 months postoperatively (Table 3).

3.2. BCVA. For NPDR without DME, one study [12] applying IVB revealed that the mean BCVA was statistically significantly better in the IVB treatment group than that in the cataract surgery alone group at 1 month postoperatively, but the mean BCVA at 3 and 6 months postoperatively had no statistically significant difference in the two groups. Three studies $[16,17,19]$ applying IVR revealed that the mean BCVA at 1 week postoperatively had no statistically significant difference in the two groups. At 1 and 3 months postoperatively, 1 study [16] reporting LogMAR visual acuity revealed that the mean BCVA had no statistically significant difference in the two groups, and 1 study [19] reporting Snellen visual acuity revealed that the mean BCVA was statistically significantly better in the IVB treatment group. At 6 months postoperatively, the mean BCVA of two studies $[16,17]$ was statistically significantly better in the IVR treatment group (Table 4).

For NPDR with DME, 3 studies [13, 14, 21] applying IVB revealed that the mean BCVA at 1 week postoperatively had no statistically significant difference in the two groups, but at 1,3 , and 6 months postoperatively, the mean BCVA was statistically significantly better in the IVB treatment group than that in the cataract surgery alone group. Two studies $[18,20]$ applying IVR revealed that the mean BCVA at 1 week and 1,3, and 6 months postoperatively was statistically significantly better in the IVR treatment group (Table 4).

Two studies $[11,15]$ reported the same inclusion criteria: CSME, NPDR, PDR, and previous focal, grid laser photocoagulation for CSME. And the postoperative laser photocoagulation was performed. Analysis of these data showed that the mean BCVA was not statistically significantly different in the both groups at 1,3 , and 6 months postoperatively (WMD: -0.05 ; 95\% CI: $-0.24,0.14 ; P=0.57$; heterogeneity: $P=0.11$, $I^{2}=60 \%$ ) (WMD: -0.04 ; 95\% CI: $-0.18,0.10$; $P=0.56$; heterogeneity: $P=0.23 ; I^{2}=30 \%$ ) (WMD: $-0.02 ; 95 \% \mathrm{CI}:-0.18$, $0.15 ; P=0.82$; heterogeneity: $\left.P=0.11 ; I^{2}=62 \%\right)$.

Analysis of all data showed that the mean BCVA at 1 week postoperatively had no statistically significant difference in the two groups, but at 1,3, and 6 months postoperatively, the mean BCVA was statistically significantly better in the anti-VEGF treatment group than that in the cataract surgery alone group (Table 4).

3.3. Postoperative Complications and Treatment. The incidence of retinopathy and maculopathy progression after cataract surgery were statistically significantly less in the intravitreal anti-VEGF treatment group $(P=0.0003$, $P<0.00001$, respectively) than those in the control group (Table 5).

The incidence of NVG progression had no statistically significant difference between both groups, but had a trend to statistical significance $(P=0.07)$ (Table 5$)$.

The rate of laser photocoagulation treatment had no statistically significant difference between both groups $(P=0.72)$ (Table 5).

There was no significant increase of intraocular pressure after surgery in each group $(P=0.21)$ (Table 5$)$.

The incidence of adverse events that were related to the injection itself, such as vitreous hemorrhage and conjunctival hemorrhage, had no statistically significant 


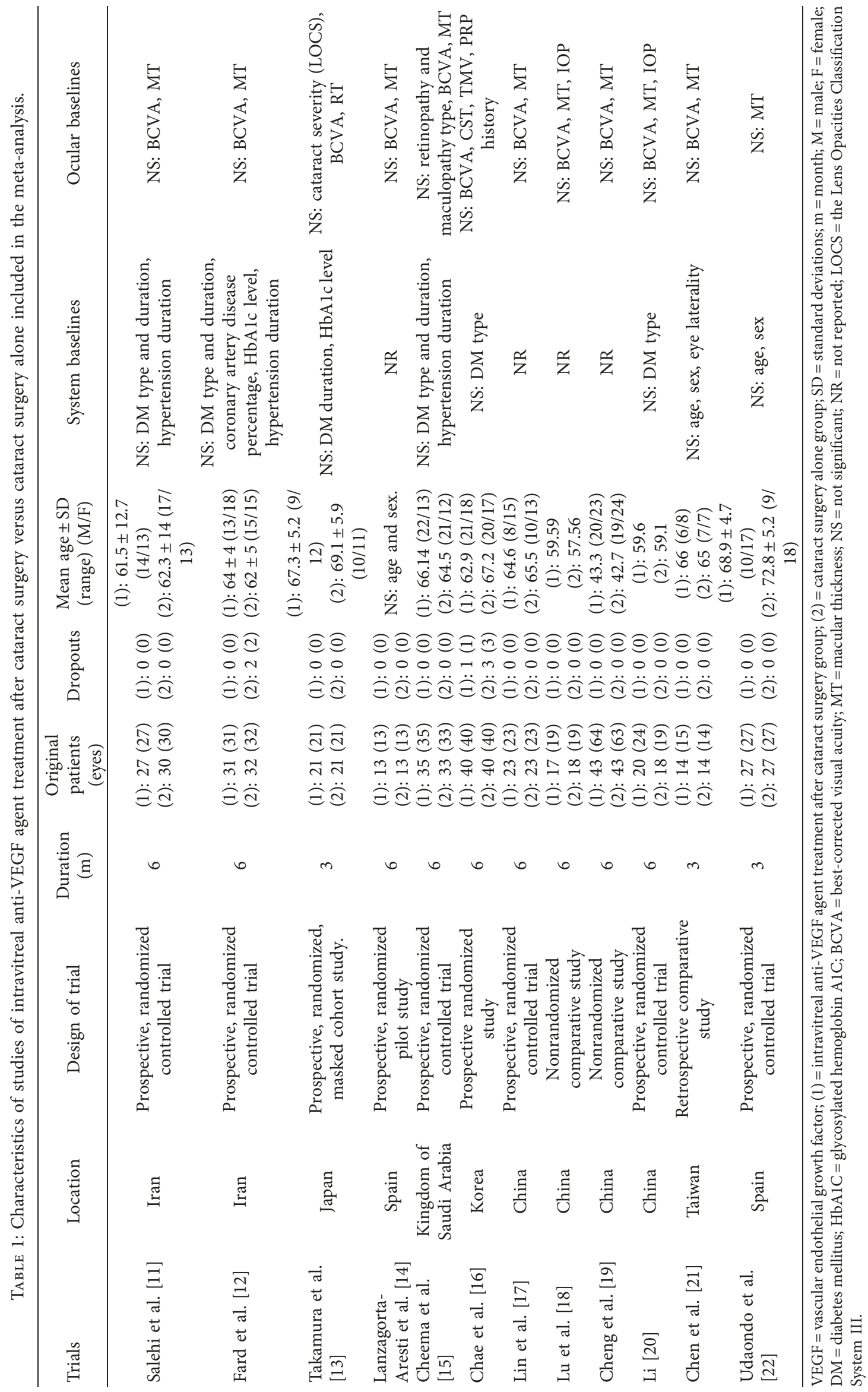




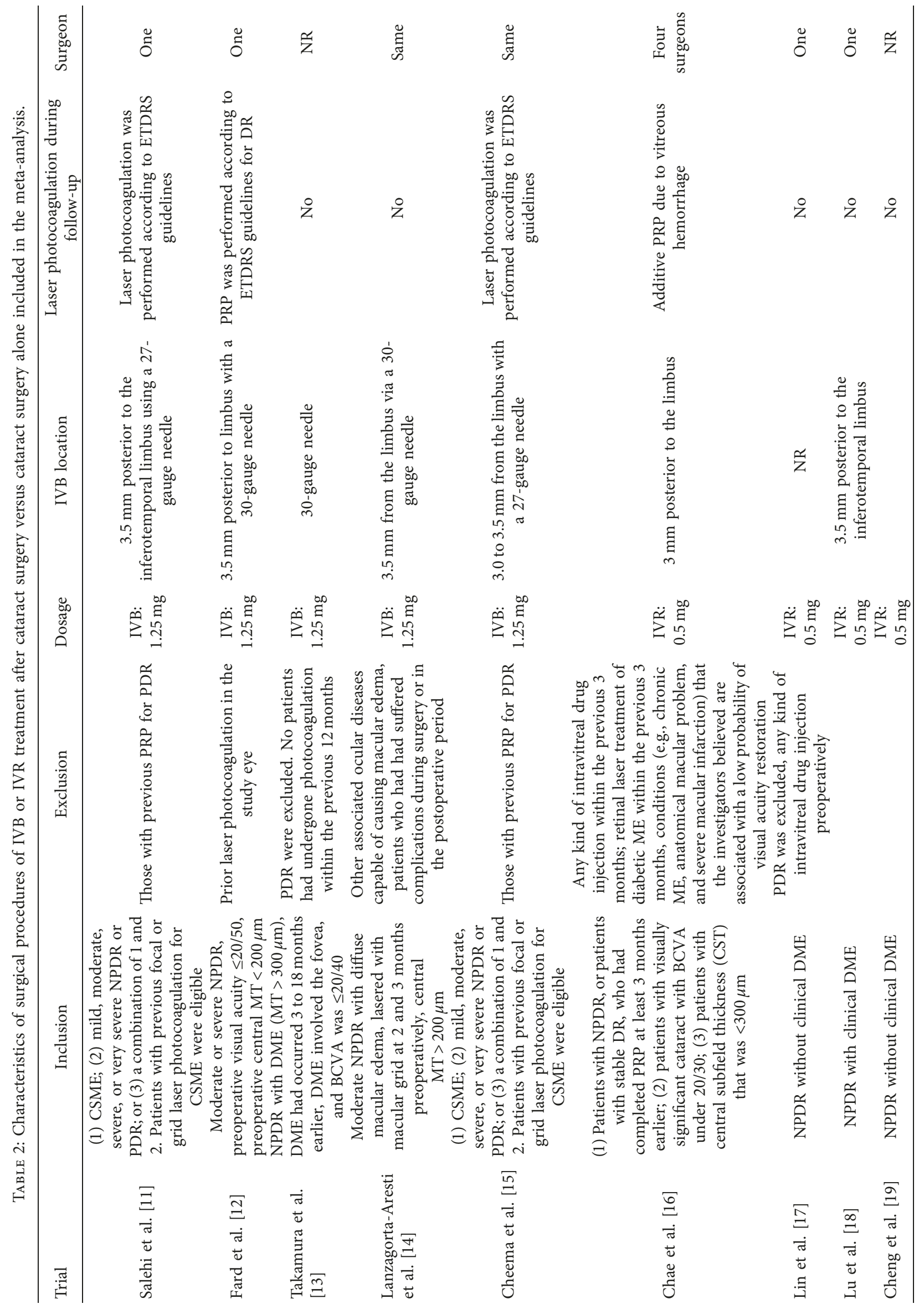




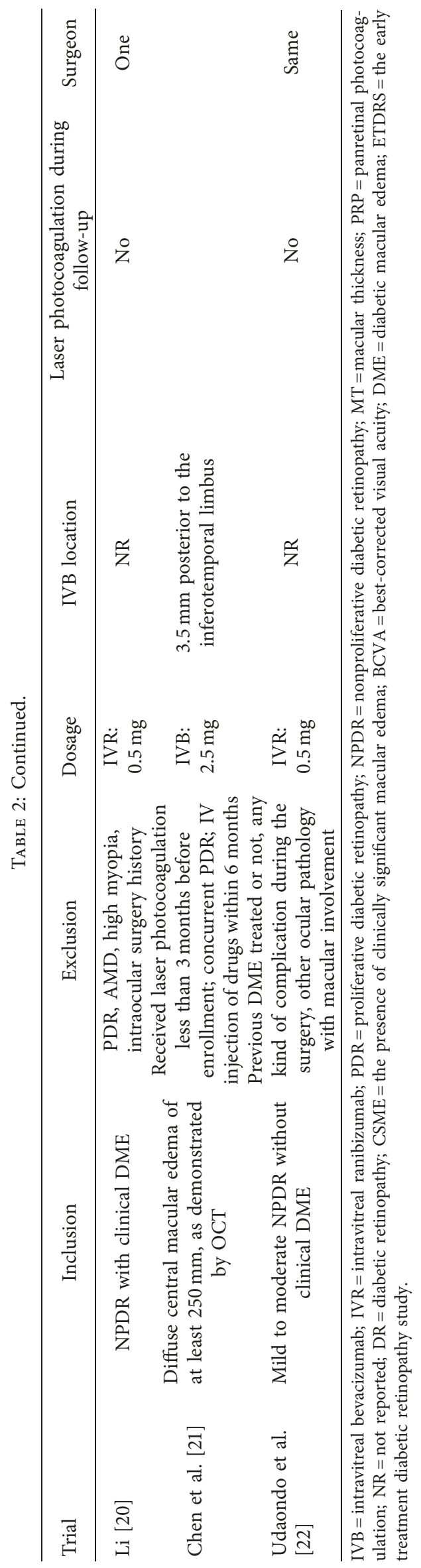


TABLE 3: Meta-analytic findings about postoperative macular thickness compared to the baseline.

\begin{tabular}{|c|c|c|c|c|c|}
\hline & No. of studies & Mean difference $(95 \% \mathrm{CI})$ & $P$ & $I^{2}(\%)$ & $\begin{array}{c}P \text { for } \\
\text { heterogeneity }\end{array}$ \\
\hline \multicolumn{6}{|l|}{ MT after 1 week } \\
\hline IVB for NPDR without DME & - & & & & \\
\hline IVR for NPDR without DME & $2[16,19]$ & $-11.69(-19.56,-3.83)$ & 0.004 & 0 & 0.80 \\
\hline IVB for NPDR with DME & - & & & & \\
\hline IVR for NPDR with DME & $1[18]$ & $-125.07(-156.34,-93.80)$ & 0.00001 & Not applicable & \\
\hline Total & $3[16,18,19]$ & $-45.95(-91.49,-0.41)$ & 0.05 & 96 & 0.00001 \\
\hline \multicolumn{6}{|l|}{ MT after 1 month } \\
\hline IVB for NPDR without DME & $1[12]$ & $-90(-105.11,-74.89)$ & 0.00001 & Not applicable & \\
\hline IVR for NPDR without DME & $3[16,19,22]$ & $-16.56(-26.10,-9.02)$ & 0.0001 & 8 & 0.34 \\
\hline IVB for NPDR with DME & $2[13,21]$ & $-84.89(-147.88,-21.91)$ & 0.008 & 63 & 0.10 \\
\hline IVR for NPDR with DME & $1[18]$ & $-153.83(-212.59,-95.07)$ & 0.00001 & Not applicable & \\
\hline Total & $9[11-13,15,16,18,19,21,22]$ & $-49.92(-78.34,-21.51)$ & 0.0006 & 93 & 0.00001 \\
\hline \multicolumn{6}{|l|}{ MT after 3 months } \\
\hline IVB for NPDR without DME & - & & & & \\
\hline IVR for NPDR without DME & $3[16,19,22]$ & $-25.64(-32.54,-18.74)$ & 0.00001 & 3 & 0.36 \\
\hline IVB for NPDR with DME & $3[13,14,21]$ & $-53.55(-72.29,-34.80)$ & 0.00001 & 0 & 0.57 \\
\hline IVR for NPDR with DME & $1[18]$ & $-108.80(-170.64,-46.96)$ & 0.0006 & Not applicable & \\
\hline Total & $9[11,13-16,18,19,21,22]$ & $-34.73(-49.94,-19.51)$ & 0.00001 & 58 & 0.01 \\
\hline \multicolumn{6}{|l|}{ MT after 6 months } \\
\hline IVB for NPDR without DME & $1[12]$ & $-24.00(-34.50,-13.50)$ & 0.00001 & Not applicable & \\
\hline IVR for NPDR without DME & $2[16,17]$ & $-24.77(-43.24,-6.30)$ & 0.009 & 0 & 0.88 \\
\hline IVB for NPDR with DME & $1[14]$ & $-109.84(-174.48,-45.20)$ & 0.0009 & Not applicable & \\
\hline IVR for NPDR with DME & $2[18,20]$ & $-90.25(-127.24,-53.26)$ & 0.00001 & 0 & 0.58 \\
\hline Total & $8[11,12,14-18,20]$ & $-45.22(-65.37,-25.07)$ & 0.00001 & 62 & 0.01 \\
\hline
\end{tabular}

$\mathrm{IVB}=$ intravitreal bevacizumab; IVR = intravitreal ranibizumab; $\mathrm{NPDR}=$ nonproliferative diabetic retinopathy; $\mathrm{MT}=$ macular thickness; $\mathrm{DME}=$ diabetic macular edema.

difference between both groups $(P=0.97, P=0.28$, respectively) (Table 5).

No adverse events, such as retinal detachment, severe ocular inflammation, endophthalmitis, or systemic adverse, were reported during the follow-up periods.

\section{Discussion}

The meta-analysis results showed that the intravitreal antiVEGF agent treatment, irrespective of IVB or IVR, immediately after cataract surgery could prevent the increase in macular thickness of eyes with NPDR without existing DME and decreasing macular thickness in NPDR with existing DME. This effect seemed to hold in 6 months postoperatively.

Similarly, the meta-analysis data also showed the intravitreal anti-VEGF agent treatment could improve better postoperative visual acuity of eyes with NPDR. For NPDR without existing DME, the effect of visual acuity improvement by IVB seemed to hold in one month postoperatively, and the effect of IVR could hold in one to 6 months postoperatively. For NPDR with existing DME, the effect of visual acuity improvement by IVB seemed to hold in 1 to 6 months postoperatively, and the effect of IVR could hold in one week to 6 months postoperatively.

Although there was no study to directly compare IVB to IVR for the treatment of diabetic macular edema immediately after cataract surgery. In the future, more trials will be needed to verify whether IVR was superior to IVB and the therapy effect of IVR was more quickly and more durable for DME after cataract surgery. Many clinical trials compared visual acuity and OCT outcomes associated with IVB vs IVR for the management of DME $[26,27]$. IVB and IVR were associated with similar effects on central macular thickness in patients with DME. IVR is associated with greater improvement in BCVA at some study visits, and the mean number of injections is higher in the IVB group.

For subgroups of eyes mixed CSME, NPDR, or PDR with no difference in postoperative macular thickness from 1 month to 3 months, the consequential effect of laser photocoagulation ought to be considered $[11,15]$. The reported rate of laser photocoagulation treatment after cataract surgery had no significant difference between the both groups $[11,15]$. This reflected that the ocular baseline especially the fundus was similar between both groups because the included studies reported that laser photocoagulation for CSME and PDR was performed promptly after cataract surgery in both groups based on ETDRS criterion. Laser photocoagulation is still a standard therapy for DME and has proven efficacy in large clinical trials $[28,29]$. So, the early macular edema or obvious macular edema was treated. Both groups had similar central macular thickness and visual acuity during the 3-month follow-up period. May be the IVB beneficial effect for macular edema was not quick. However, the 6-month data revealed that the IVB treatment still had a little beneficial effect in preventing the macular thickening.

Although the treatment rate of laser photocoagulation had no significant difference, we thought the efficacy of laser photocoagulation after the intravitreous pretreatment group was high. As Lang's study revealed, intravitreal anti-VEGF agent treatment plus laser has also proven to be more 
TABLE 4: Meta-analytic findings about postoperative best-corrected visual acuity compared to the baseline.

\begin{tabular}{|c|c|c|c|c|c|}
\hline & No. of studies & Mean difference $(95 \% \mathrm{CI})$ & $P$ & $I^{2}(\%)$ & $\begin{array}{c}P \text { for } \\
\text { heterogeneity }\end{array}$ \\
\hline \multicolumn{6}{|l|}{ BCVA after 1 week } \\
\hline IVR for NPDR without DME (LogMAR) & $1[16]$ & $-0.06(-0.18,0.06)$ & 0.32 & Not applicable & \\
\hline IVR for NPDR without DME (Snellen) & $2[17,19]$ & $0.04(-0.04,0.12)$ & 0.35 & 50 & 0.16 \\
\hline IVB for NPDR with DME (LogMAR) & $1[21]$ & $-0.13(-0.51,0.25)$ & 0.50 & Not applicable & \\
\hline IVR for NPDR with DME (Snellen) & $1[18]$ & $0.16(0.02,0.30)$ & 0.02 & Not applicable & \\
\hline Total: (LogMAR) & $2[16,21]$ & $-0.07(-0.18,0.05)$ & 0.25 & 0 & 0.73 \\
\hline Total: (Snellen) & $3[17-19]$ & $0.06(-0.01,0.14)$ & 0.11 & 51 & 0.13 \\
\hline \multicolumn{6}{|l|}{ BCVA after 1 month } \\
\hline IVB for NPDR without DME (LogMAR) & $1[12]$ & $-0.24(-0.34,-0.14)$ & 0.00001 & Not applicable & \\
\hline IVR for NPDR without DME (LogMAR) & $1[16]$ & $-0.07(-0.17,0.03)$ & 0.15 & Not applicable & \\
\hline IVR for NPDR without DME (Snellen) & $1[19]$ & $0.15(0.09,0.21)$ & 0.00001 & Not applicable & \\
\hline IVB for NPDR with DME (LogMAR) & $2[13,21]$ & $-0.20(-0.37,-0.03)$ & 0.02 & 0 & 0.75 \\
\hline IVR for NPDR with DME (Snellen) & $1[18]$ & $0.19(0.01,0.37)$ & 0.04 & Not applicable & \\
\hline Total: (LogMAR) & $6[11-13,15,16,21]$ & $-0.14(-0.19,-0.09)$ & 0.0001 & 50 & 0.07 \\
\hline Total: (Snellen) & $2[18,19]$ & $0.15(0.10,0.21)$ & 0.00001 & 0 & 0.68 \\
\hline \multicolumn{6}{|l|}{ BCVA after 3 months } \\
\hline IVB for NPDR without DME (LogMAR) & $1[12]$ & $-0.04(-0.14,0.06)$ & 0.41 & Not applicable & \\
\hline IVR for NPDR without DME (LogMAR) & $1[16]$ & $-0.03(-0.12,0.06)$ & 0.50 & Not applicable & \\
\hline IVR for NPDR without DME (Snellen) & $1[19]$ & $0.20(0.15,0.25)$ & 0.00001 & Not applicable & \\
\hline IVB for NPDR with DME (LogMAR) & $2[13,21]$ & $-0.13(-0.24,-0.02)$ & 0.02 & 0 & 0.91 \\
\hline IVB for NPDR with DME (Snellen) & $1[14]$ & $0.19(0.02,0.36)$ & 0.03 & Not applicable & \\
\hline IVR for NPDR with DME (Snellen) & $1[18]$ & $0.23(0.05,0.41)$ & 0.01 & Not applicable & \\
\hline Total: (LogMAR) & $6[11-13,15,16,21]$ & $-0.06(-0.11,-0.01)$ & 0.02 & 0 & 0.58 \\
\hline Total: (Snellen) & $3[14,18,19]$ & $0.20(0.16,0.25)$ & 0.00001 & 0 & 0.94 \\
\hline \multicolumn{6}{|l|}{ BCVA after 6 months } \\
\hline IVB for NPDR without DME (LogMAR) & $1[12]$ & $0.00(-0.10,0.10)$ & 1.00 & Not applicable & \\
\hline IVR for NPDR without DME (LogMAR) & $1[16]$ & $-0.13(-0.24,-0.02)$ & 0.02 & Not applicable & \\
\hline IVR for NPDR without DME (Snellen) & $1[17]$ & $0.15(0.05,0.25)$ & 0.005 & Not applicable & \\
\hline IVB for NPDR with DME (Snellen) & $1[14]$ & $0.26(0.10,0.42)$ & 0.002 & Not applicable & \\
\hline IVR for NPDR with DME (Snellen) & $2[18,20]$ & $0.19(0.10,0.28)$ & 0.00001 & 0 & 1.00 \\
\hline Total: (LogMAR) & $4[11,12,15,16]$ & $-0.07(-0.11,-0.02)$ & 0.006 & 21 & 0.29 \\
\hline Total: (Snellen) & $4[14,17,18,20]$ & $0.19(0.12,0.25)$ & 0.00001 & 0 & 0.74 \\
\hline
\end{tabular}

$\mathrm{IVB}=$ intravitreal bevacizumab; IVR = intravitreal ranibizumab; NPDR=nonproliferative diabetic retinopathy; BCVA=best-corrected visual acuity; DME $=$ diabetic macular edema.

TABle 5: Postoperative events and treatment in the meta-analysis.

\begin{tabular}{lccccccc}
\hline Events & No. of studies & $\begin{array}{c}\text { Crude rate, } n / N \\
\text { Intervention }\end{array}$ & Control & $\begin{array}{c}\text { Rate difference } \% \\
(95 \% \text { CI })\end{array}$ & $\begin{array}{c}P \text { for overall } \\
\text { effect }\end{array}$ & $\begin{array}{c}I^{2} \text { for heterogeneity } \\
(\%)\end{array}$ & $\begin{array}{c}P \text { for } \\
\text { heterogeneity }\end{array}$ \\
\hline $\begin{array}{l}\text { DR progression } \\
\text { Maculopathy }\end{array}$ & $3[1,2,5]$ & $12 / 93$ & $34 / 93$ & $0.26[0.13,0.54]$ & 0.0003 & 27 & 0.26 \\
progression & $3[1,5,6,12]$ & $18 / 128$ & $68 / 127$ & $0.10[0.05,0.20]$ & $<0.00001$ & 0 & 0.81 \\
NVG progression & $2[1,5]$ & $1 / 62$ & $7 / 63$ & $0.19[0.03,1.13]$ & 0.07 & 0 & 0.97 \\
Laser photocoagulation & $4[1,2,5,6]$ & $36 / 132$ & $34 / 130$ & $1.13[0.59,2.13]$ & 0.72 & - & 0.86 \\
VH & $1[6]$ & $1 / 39$ & $1 / 37$ & $0.95[0.06,15.72]$ & 0.97 & - \\
Elevated IOP & $2[7,9]$ & $3 / 87$ & $0 / 86$ & $4.12[0.45,37.82]$ & 0.21 & 0.83 \\
CH & $1[7]$ & $2 / 23$ & $0 / 23$ & $5.47[0.25$, & 0.28 & - & - \\
\hline
\end{tabular}

$\mathrm{IVB}=$ intravitreal bevacizumab; $\mathrm{DR}=$ diabetic retinopathy; $\mathrm{NVG}=$ neovascular glaucoma; $\mathrm{CI}=$ confidence interval; $\mathrm{VH}=\mathrm{vitreous}$ hemorrhage; $\mathrm{CH}=$ conjunctival hemorrhage.

effective for the treatment of PDR compared to laser alone [30]. It can improve the resolution of vitreous and retinal hemorrhage and facilitate laser photocoagulation completion. So, the completely laser therapy significantly would reduce the incidence of progression of retinopathy and maculopathy in eyes that received intravitreal anti-VEGF agent treatment. This is a synergistic prophylactic effect determined by laser therapy and intravitreal anti-VEGF agent treatment. Although the incidence of progression of NVG had no significant difference in both groups, the incidence (7/63) of progression of NVG in eyes without receiving intravitreal anti-VEGF agent treatment was more times higher than those $(1 / 62)$ with receiving treatment, and there was a trend to statistically significant difference. 
Many studies have been published to prove whether cataract surgery itself or other factors increase the risk of DR progression postoperatively $[31,32]$. Whatever reasons, cataract surgery must be performed for some diabetic patients. And how to prevent postoperatively macular thickening and retinopathy progression is the main question. Besides the beneficial effect of intravitreal antiVEGF agent treatment, the traditional treatment still yielded better results in terms of macular thickness reduction and improvement of VA, such as laser photocoagulation, intravitreal triamcinolone, or dexamethasone implant treatment [7, 33-35], but intravitreal triamcinolone treatment was associated with a greater risk of intraocular pressure elevation. An optional therapy was determined by not only the better clinical outcomes but also the fewer side effects. The selected trials revealed that intravitreal anti-VEGF agent treatment was well tolerated, and no local and systemic adverse events were noticed during the study. However, from the values of macular thickness during follow-up, there was a trend of macular thickening from 3 to 6 months postoperatively $[15,16]$. The drawback of its nonpermanent effect and higher costs ought to be of concern [36]. Further therapy for diabetic retinopathy after cataract surgery was integrated by different treatment methods according to their respective advantages.

This present meta-analysis might have some limitations. One weakness of our study was that there was no trial exclusively focused on PDR as subgroup analysis. The metaanalysis results should be accurate and comprehensive if adding the subgroup analysis of PDR with or without macular edema. Secondly, the studies included were heterogeneous in terms of study location, population, number of patients from different studies and basal condition, and study quality (relative methodological strengths and weaknesses). The strengths of this study include the randomized study design and nonrandomized comparative study design. The baseline conditions in these nonrandomized comparative studies were highly controlled. So it is feasible to include these studies to perform meta-analysis. More study participants were included to research, and the results were more closed to be representative of the real-world population with DME. These findings still need to be interpreted with caution. Bevacizumab is an off-label anti-VEGF agent for DME or postsurgical macular edema. It must be used with caution.

In conclusion, the present study demonstrated that intravitreal anti-VEGF agent treatment plus cataract surgery may represent a safe and effective strategy to prevent postoperative macular thickening or decreasing macular edema and result in greater mean improvements in visual acuity for diabetic patients. In future, new treatments, such as other anti-VEGF agent injection and intravitreal anti-VEGF agents combined subtenon triamcinolone injection, should be investigated to improve clinical efficacy, and high-quality, largescale, multicenter randomized control trials will be needed to verify [37]. The optimized therapy should be suggested when using the data from the evidence-based study to guide treatment considerations for an individual patient.

\section{Conflicts of Interest}

The authors declare that they have no conflicts of interest.

\section{References}

[1] S. J. Kim, R. Equi, and N. M. Bressler, "Analysis of macular edema after cataract surgery in patients with diabetes using optical coherence tomography," Ophthalmology, vol. 114, no. 5, pp. 881-889, 2007.

[2] D. Squirrell, R. Bhola, J. Bush et al., "A prospective, case controlled study of the natural history of diabetic retinopathy and maculopathy after uncomplicated phacoemulsification cataract surgery in patients with type 2 diabetes," British Journal of Ophthalmology, vol. 86, no. 5, pp. 565-571, 2002.

[3] N. Dong, B. Xu, and B. Wang, "Aqueous cytokines as predictors of macular edema in patients with diabetes following uncomplicated phacoemulsification cataract surgery," BioMed Research International, vol. 2015, Article ID 126984, 8 pages, 2015.

[4] J. I. Patel, P. G. Hykin, and I. A. Cree, "Diabetic cataract removal: postoperative progression of maculopathy-growth factor and clinical analysis," British Journal of Ophthalmology, vol. 90, no. 6, pp. 697-698, 2006.

[5] M. E. Hartnett, N. Tinkham, L. Paynter et al., "Aqueous vascular endothelial growth factor as a predictor of macular thickening following cataract surgery in patients with diabetes mellitus," American Journal of Ophthalmology, vol. 148, no. 6, pp. 895-901, 2009.

[6] H. Funatsu, H. Yamashita, H. Noma, E. Shimizu, T. Mimura, and S. Hori, "Prediction of macular edema exacerbation after phacoemulsification in patients with nonproliferative diabetic retinopathy," Journal of Cataract \& Refractive Surgery, vol. 28, no. 8, pp. 1355-1363, 2002.

[7] L. L. Lim, J. L. Morrison, M. Constantinou et al., "Diabetic macular edema at the time of cataract surgery trial: a prospective, randomized clinical trial of intravitreous bevacizumab versus triamcinolone in patients with diabetic macular oedema at the time of cataract surgery-preliminary 6 month results," Clinical \& Experimental Ophthalmology, vol. 44, no. 4, pp. 233-242, 2016.

[8] I. Zechmeister-Koss and M. Huić, "Vascular endothelial growth factor inhibitors (anti-VEGF) in the management of diabetic macular oedema: a systematic review," British Journal of Ophthalmology, vol. 96, no. 2, pp. 167-178, 2012.

[9] J. A. Wells, A. R. Glassman, A. R. Ayala et al., "Aflibercept, bevacizumab, or ranibizumab for diabetic macular edema," Ophthalmology, vol. 123, no. 6, pp. 1351-1359, 2016.

[10] G. Virgili, M. Parravano, J. R. Evans, I. Gordon, and E. Lucenteforte, "Anti-vascular endothelial growth factor for diabetic macular oedema: a network meta-analysis," Cochrane Database of Systematic Reviews, vol. 6, article CD007419, 2017.

[11] A. Salehi, A. N. Beni, H. Razmjoo, and Z. N. Beni, "Phacoemulcification with intravitreal Bevacizumab injection in patients with cataract and coexisting diabetic retinopathy: prospective randomized study," Journal of Ocular Pharmacology and Therapeutics, vol. 28, no. 3, pp. 212-218, 2012.

[12] M. A. Fard, A. Y. Abyane, and M. Malihi, "Prophylactic intravitreal Bevacizumab for diabetic macular edema (thickening) after cataract surgery: prospective randomized study," European Journal of Ophthalmology, vol. 21, no. 3, pp. 276-281, 2011.

[13] Y. Takamura, E. Kubo, and Y. Akagi, "Analysis of the effect of intravitreal Bevacizumab injection on diabetic macular edema 
after cataract surgery," Ophthalmology, vol. 116, no. 6, pp. 1151-1157, 2009.

[14] A. Lanzagorta-Aresti, E. Palacios-Pozo, J. L. Menezo Rozalen, and A. Navea-tejerina, "Prevention of vision loss after cataract surgery in diabetic macular edema with intravitreal bevacizumab," Retina, vol. 29, no. 4, pp. 530-535, 2009.

[15] R. A. Cheema, M. M. Al-Mubarak, Y. M. Amin, and M. A. Cheema, "Role of combined cataract surgery and intravitreal bevacizumab injection in preventing progression of diabetic retinopathy," Journal of Cataract \& Refractive Surgery, vol. 35, no. 1, pp. 18-25, 2009.

[16] J. B. Chae, S. G. Joe, S. J. Yang et al., "Effect of combined cataract surgery and Ranibizumab injection in postoperative macular edema in nonproliferative diabetic retinopathy," Retina, vol. 34, no. 1, pp. 149-156, 2014.

[17] W. H. Lin, M. Lu, H. Y. Tang, and Z. R. Zeng, "Clinical application of Ranibizumab in the therapy of diabetic cataract," Guoji Yanke Zazhi, vol. 15, pp. 880-882, 2015.

[18] Y. Lu, Q. Fan, L. Yang, and Y. Dong, "Effect of combined senile diabetic cataract surgery and intravitreal lucentis injection on diabetic macular edema," Chinese Journal of Practical Ophthalmology, vol. 32, pp. 560-563, 2014.

[19] M. Z. Cheng, C. Wang, and M. Chen, "Phacoemulsification combined with intravitreal injection of lucentis for diabetic cataract," Recent Advances in Ophthalmology, vol. 36, pp. 754-756, 2016.

[20] T. Li, "The effect of senile cataract surgery combined with vitreous cavity injection of monoclonal antibody on diabetic macular edema," China Continuing Medical Education, vol. 8, pp. 149-151, 2016.

[21] C.-H. Chen, Y.-C. Liu, and P.-C. Wu, "The combination of intravitreal bevacizumab and phacoemulsification surgery in patients with cataract and coexisting diabetic macular edema," Journal of Ocular Pharmacology and Therapeutics, vol. 25, no. 1, pp. 83-90, 2009.

[22] P. Udaondo, M. Garcia-Pous, S. Garcia-Delpech, D. Salom, and M. Diaz-Llopis, "Prophylaxis of macular edema with intravitreal ranibizumab in patients with diabetic retinopathy after cataract surgery: a pilot study," Journal of Ophthalmology, vol. 2011, Article ID 159436, 4 pages, 2011.

[23] M. Egger, G. D. Smith, and A. N. Phillips, "Meta-analysis: principles and procedures," BMJ, vol. 315, no. 7121, pp. 1533-1534, 1997.

[24] L.-Q. Zhao, H. Zhu, P.-Q. Zhao, and Y.-Q. Hu, “A systematic review and meta-analysis of clinical outcomes of vitrectomy with or without intravitreal Bevacizumab pretreatment for severe diabetic retinopathy," British Journal of Ophthalmology, vol. 95, no. 9, pp. 1216-1222, 2011.

[25] D. O'Connor, S. Green, and J. P. Higgins, "Defining the review question and developing criteria for including studies," in Cochrane Handbook for Systematic Reviews of Interventions, Cochrane Collaboration, Oxford, UK, 2008.

[26] A. B. Nepomuceno, E. Takaki, F. P. Paes de Almeida et al., "A prospective randomized trial of intravitreal bevacizumab versus ranibizumab for the management of diabetic macular edema," American Journal of Ophthalmology, vol. 156, no. 3, pp. 502-510, 2013.

[27] H. E. Wiley, D. J. S. Thompson, C. Bailey et al., "A crossover design for comparative efficacy," Ophthalmology, vol. 123, no. 4, pp. 841-849, 2016.

[28] D. S. Fong, S. F. Strauber, L. P. Aiello et al., "Comparison of the modified early treatment diabetic retinopathy study and mild macular grid laser photocoagulation strategies for diabetic macular edema," Archives of Ophthalmology, vol. 125, pp. 469-480, 2007.

[29] Diabetic Retinopathy Clinical Research Network, "A randomized trial comparing intravitreal triamcinolone acetonide and focal/grid photocoagulation for diabetic macular edema," Ophthalmology, vol. 115, pp. 1447-1449, 2008.

[30] G. E. Lang, S. Liakopoulos, J. Vögeler et al., "The relation study: efficacy and safety of ranibizumab combined with laser photocoagulation treatment versus laser monotherapy in NPDR and PDR patients with diabetic macular oedema," Acta Ophthalmologica, vol. 96, no. 3, pp. e377-e385, 2018.

[31] J. Dowler, K. S. Sehmi, P. G. Hykin et al., "The natural history of macular edema after cataract surgery in diabetes," Ophthalmology, vol. 106, no. 4, pp. 663-668, 1999.

[32] T. Hong, P. Mitchell, T. de Loryn, E. Rochtchina, S. Cugati, and J. J. Wang, "Development and progression of diabetic retinopathy 12 months after phacoemulsification cataract surgery," Ophthalmology, vol. 116, no. 8, pp. 1510-1514, 2009.

[33] D. L. C. Isaac, M. B. Abud, K. A. Frantz, A. R. Rassi, and M. Avila, "Comparing intravitreal triamcinolone acetonide and bevacizumab injections for the treatment of diabetic macular oedema: a randomized double-blind study," Acta Ophthalmologica, vol. 90, no. 1, pp. 56-60, 2012.

[34] G. Sobaci, G. Özge, C. Erdurman, H. A. Durukan, and Z. M. Bayraktar, "Comparison of grid laser, intravitreal triamcinolone, and intravitreal Bevacizumab in the treatment of diffuse diabetic macular edema," Ophthalmologica, vol. 227, no. 2, pp. 95-99, 2012.

[35] A. M. Sze, F. O. Luk, T. P. Yip, G. K. Lee, and C. K. Chan, "Use of intravitreal dexamethasone implant in patients with cataract and macular edema undergoing phacoemulsification," European Journal of Ophthalmology, vol. 25, no. 2, pp. 168172, 2015.

[36] D. E. Welch, H. Elmariah, M. C. Peden, S. G. Adams, R. Ratnakaram, and S. Kaushal, "Short-term response of macular oedema to intravitreal bevacizumab," British Journal of Ophthalmology, vol. 93, no. 8, pp. 1033-1036, 2009.

[37] S.-Y. Yu, D. H. Nam, and D. Y. Lee, "Changes in aqueous concentrations of various cytokines after intravitreal bevacizumab and subtenon triamcinolone injection for diabetic macular edema," Graefe's Archive for Clinical and Experimental Ophthalmology, vol. 256, no. 1, pp. 39-47, 2018. 


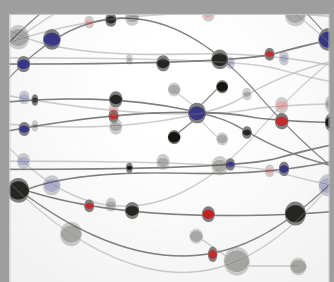

The Scientific World Journal


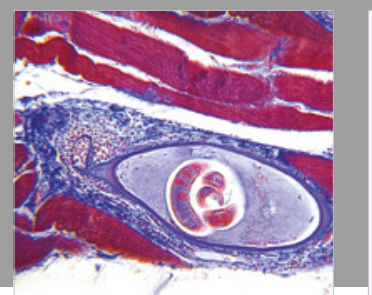

Gastroenterology Research and Practice

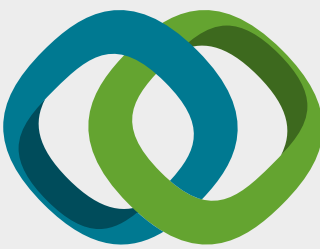

\section{Hindawi}

Submit your manuscripts at

www.hindawi.com


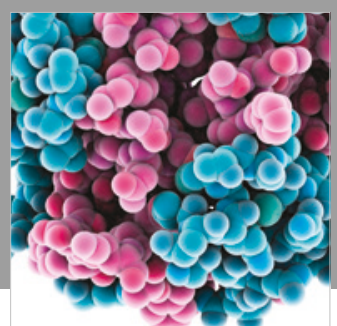

ournal of

Diabetes Research

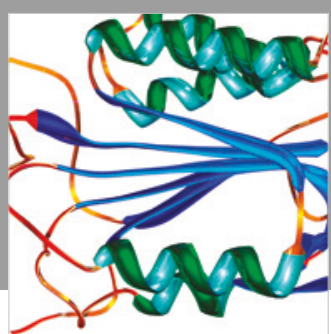

Disease Markers
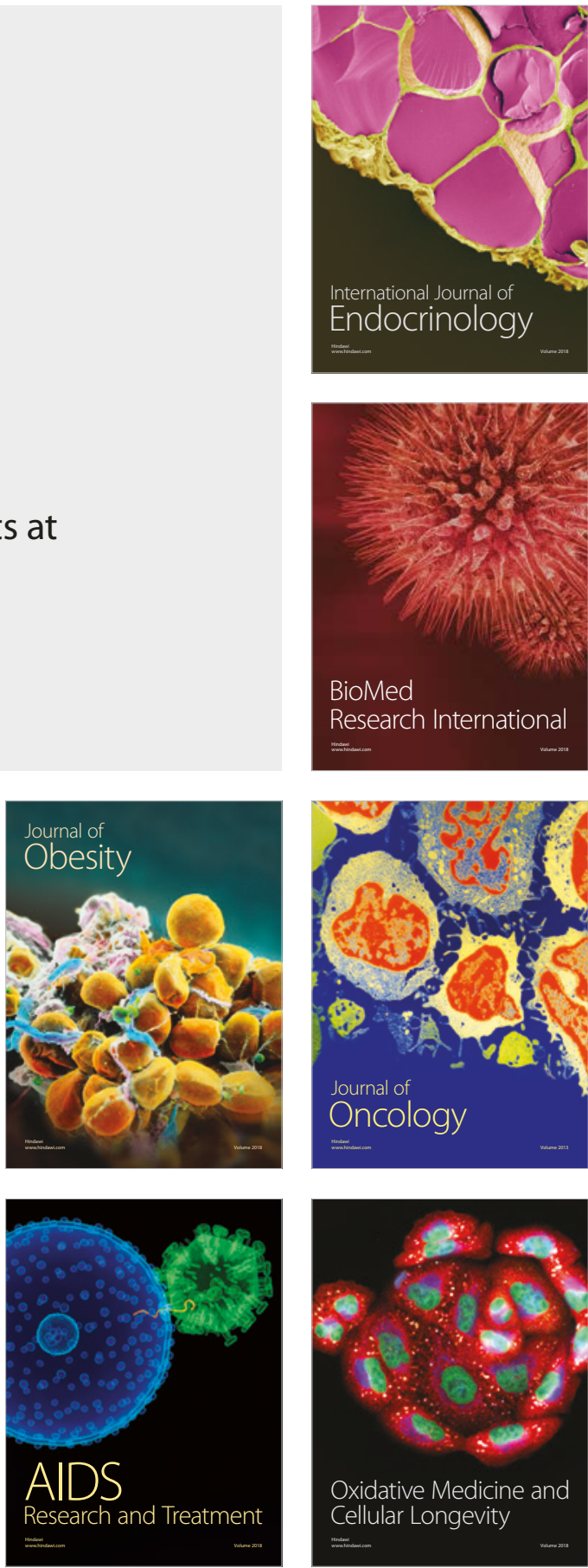